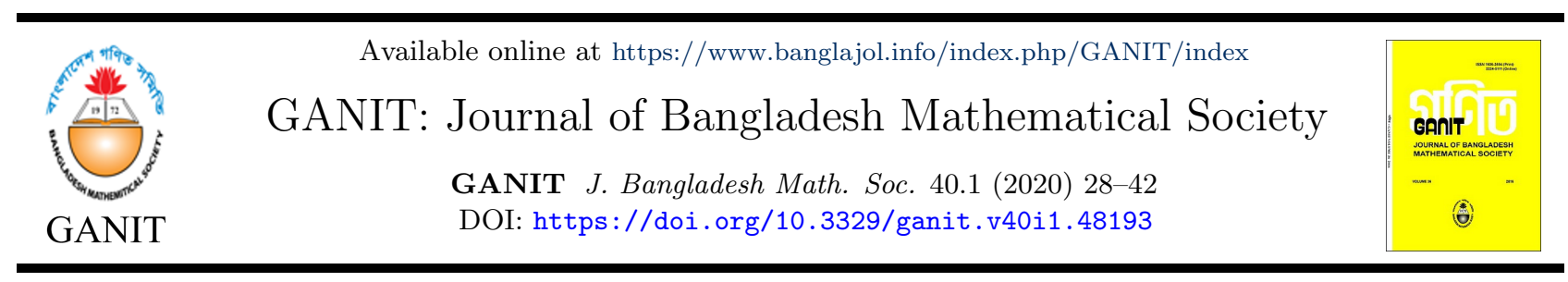

\title{
Global Spectral Collocation Method with Fourier Transform to Solve Differential Equations
}

\author{
Sayeda Irin Akter ${ }^{\mathrm{a}}$, MD. Shahriar Mahmud ${ }^{\mathrm{b}}$, Md. Kamrujjaman *c ${ }^{*}$, and Hazrat Ali ${ }^{\mathrm{d}}$ \\ ${ }^{\mathrm{a}, \mathrm{b}, \mathrm{c}}$ Department of Mathematics, University of Dhaka, Dhaka 1000, Bangladesh \\ d Department of Applied Mathematics, University of Dhaka, Dhaka 1000, Bangladesh
}

\begin{abstract}
Numerical analysis is the area of mathematics that creates, analyzes, and implements algorithms for solving numerically the problems from real-world applications of algebra, geometry, and calculus, and they involve variables which vary continuously. Till now, numerous numerical methods have been introduced. Spectral method is one of those techniques used in applied mathematics and scientific computing to numerically solve certain differential equations, potentially involving the use of the Fast Fourier Transform (FFT). This study presents some of the fundamental ideas of spectral method. Orthogonal basis are used to establish computational algorithm. The accuracy and efficiency of proposed model are discussed. This manuscript estimates for the error between the exact and approximated discrete solutions. This paper shows that, grid points for polynomial spectral methods should lie approximately in a minimal energy configuration associated with inverse linear repulsion between points. The wave equation, linear and non-linear boundary value problems are solved using spectral method on the platform of MATLAB language.
\end{abstract}

(C) 2020 Published by Bangladesh Mathematical Society Received: 29 March, 2020

Accepted: 09 July, 2020

Keywords: Spectral method; Differential equations; Basis function; Chebyshev polynomial; Fast Fourier Transformation; Eigenfunctions.

AMS Subject Classification 2010: 74S25 - 33F05 - 34K28 - 46N40 - 65T50 - 41A50.

\section{Introduction}

In 1977 Gottlieb and Orszag reviewed the state of the art in the monograph, at that time, of spectral methods. The theory and applications presented in that work focused on the tau and Galerkin methods confined the fluid dynamical applications. In the past few decades, there has been extensive activity both in the theory and application of spectral methods; mainly concentrated in the area of pseudospectral methods. In case of theory, functional analysis has proved to be a powerful tool for obtaining useful error estimates. For spectral techniques, improved iteration methods has been developed that make possible large-scale calculations of complicated physical phenomena [1]. Some common areas of applications are [1,2]

- compressible flow problems and rather complicated flow fields

${ }^{*}$ Corresponding author: M. Kamrujjaman, E-mail address: kamrujjaman@du.ac.bd 
- shock waves and significant progress on transition and turbulence flows

- applied mathematics and scientific computing to numerically solve certain differential equations, potentially involving the use of the FFT

- in kinetic theory, and

- spectral analysis of signals.

Our goal in this study is to introduce the potential use of applications of spectral methods and to the problems encountered in implementing them. Spectral methods provide a very sophisticated tool for scientific computing; as in the case of other sophisticated techniques. A properly constructed spectral method can be used to obtain solutions where other numerical techniques fail.

Spectral method is a method of great consequence in solving differential equations numerically arises in applied mathematics and engineering, potentially involving the use of the Fast Fourier Transform [2, 3, 4]. The main feature of this method is to express the differential equation as a sum of some basis functions with suitable coefficients that satisfy the differential equation $[3,5]$.

The idea of the spectral method and the Galerkin Finite Element Method (GFEM) are analogous except that its used basis functions are non-zero over the whole domain where GFEM uses basis functions that are non-zero only on small subdomains $[6,7,8]$. That is, the approach of the spectral method is global where the GFEM is local [7]. The spectral method is an efficient method with less computational cost and higher accuracy than GFEM for solving ordinary differential equations (ODEs), partial differential equations (PDEs) and eigenvalue problems with differential equations except some special cases such as complex geometries and discontinuous coefficients $[2,9,10]$. In this paper, we have studied the spectral methods via Fast Fourier Transformation, Chebyshev points and using Chebyshev differentiation matrix to find the higher accuracy of smooth functions and to solve the boundary value problems with homogeneous and non-homogeneous dirichlet boundary condition's.

The main novelties of this paper are described as follows:

i. We choose the basis function and use the Fast Fourier Transformation for periodic bounded grid.

ii. We take Chebyshev points and using Chebyshev differentiation matrix to solve problems for non-periodic grid.

iii. Using spectral methods, we have solved the boundary value problems; for example the wave equation, linear \& non-linear boundary value problems.

iv. To validate and consistency of the method, we also solved the linear and non-linear boundary value problems using finite element method (FEM).

v. Besides the efficiency and significance of spectral methods, we have listed out the limitations of the method.

It is found that the accuracy of the results using spectral method is incompatible than any other robust numerical methods.

This paper is organized in the following manner.

Section 2 contains all the necessary preliminary ideas about the spectral collocation method including Fast and Discrete Fourier Transform. The next section 3 discusses about Chebyshev points with reference figures to understand. The accuracy of this underlining method is studied in section 4 . This section studies the uniqueness, time and space discretization for the method. Subsections 4.4, 4.5 discuss about the error estimation and accuracy using Fourier Transform respectively according to the spectral collocation methods. In section 5 we show some examples using the prescribed method. The last section 6 concludes with the discussion remarks.

\section{Preliminaries}

Let, $\Omega \subset R^{d}, d=2,3, \ldots$ be a bounded connected domain, with boundary $\Gamma=\partial \Omega$ and let $T$ be a positive real number. We consider the heat equation [4]

$$
\begin{cases}u_{t}-\Delta u=f, & \text { in } \Omega \times] 0, T[ \\ u=0, & \text { on } \partial \Omega \times] 0, T[ \\ u(\cdot, 0)=u_{0}, & \text { in } \Omega .\end{cases}
$$


Where $f \in L^{2}(\Omega \times] 0, T[)$ and $u_{0} \in L^{2}(\Omega)$ are given and $u$ is the unknown function. For the bounded periodic problems the basis functions consist of trigonometric polynomials of the form

$$
u_{N}(x, t)=\alpha_{0}(t)+\sum_{i=1}^{N}\left[\alpha_{i}(t) \cos (k \pi x)+\beta_{i}(t) \sin (k \pi x)\right]
$$

This basis function satisfies the requirements

i. The approximations $u_{N}(x, t)$ should converge rapidly to $u(x, t)$ as $n \rightarrow \infty$.

ii. For all the given coefficients $\left\{\alpha_{i}(t)\right\}, i=0,1,2, \ldots, N$; it should be easy to determine another set of coefficients $\left\{\alpha_{i}^{\prime}(t)\right\}, i=0,1,2, \ldots, N$ such that

$$
\frac{\partial u_{N}}{\partial x}=\sum_{i=0}^{N} \alpha_{i}(t) \frac{\mathrm{d} \psi_{i}(x)}{\mathrm{d} x} \rightsquigarrow \sum_{i=0}^{N} \alpha_{i}^{\prime}(t) \psi_{i}(x) .
$$

iii. The computation of expansion coefficients $\left\{\alpha_{i}(t)\right\}, i=0,1,2, \ldots, N$; from function values $\left(x_{i}, t\right), i=$ $0,1, \ldots, N$; should be easy. That is, the conversion between the two data sets is algorithmically efficient

$$
\left\{u\left(x_{i}, t\right)_{i=0}^{N}\right\} \rightleftarrows\left\{\alpha_{i}\right\}_{i=0}^{N}
$$

If $N$ is highly composite, FFT enables us to compute the Discrete Fourier Transformation (DFT) and hence spectral derivatives in $O(N \log N)$ floating point operations. The FFT rapidly computes such transformations by factorizing the (DFT) matrix into a product of sparse factors. As a result, it manages to reduce the complexity of computing the DFT from $O\left(N^{2}\right)$ to $O(N \log N)$. Here the algorithm is to breaks down a DFT of any composite size $N=N_{1} N_{2}$ into many smaller DFT's of sizes $N_{1}$ and $N_{2}$, along with $O(N)$ multiplications by complex roots of unity.

The data $u$ to be differentiated will be real. To construct our numerical scheme, we proceed just as we might with a finite difference approximation of a PDE. For the time derivative we use a leap frog formula and we approximate the derivative spectrally. Let $v^{(n)}$ be the vector at time step $n$ that approximates $u\left(x_{j}, n \Delta t\right)$. At grid point $x_{j}$ our spectral derivative is $D v^{(n)}$, where $D$ is the spectral differentiation matrix, which we implement by the FFT. Here the approximation becomes

$$
v_{j}^{(n+1)}-v_{j}^{(n-1)}=-2 \Delta t c\left(x_{j}\right)\left(D v^{(n)}\right)_{j} ; \quad j=1,2,3, \ldots, N .
$$

The leap frog scheme requires two initial conditions to start where the given PDE provides only one. To obtain a starting value $v^{(n)}$ one can use one-step ODE formula, such as Runge-Kutta formula [11], to generate the second set of initial data.

Definition 1. [3, 4] A hat function is a function whose graph takes the shape of a hat or a triangle. Often this is an isosceles triangle of height 1 and base 2 in which case it is referred to as the triangular function. The most common definition is as a piecewise function:

$$
\begin{aligned}
\Lambda(x) & =\max (1-|x|, 0) \\
& =\left\{\begin{array}{l}
1-|x|,|x|<1 \\
0, \quad \text { Otherwise }
\end{array}\right.
\end{aligned}
$$

Definition 2. [3, 4] A smooth function is a function that has continuous derivatives up to some desired order over some domain. If the graph of the function's derivative also contains no breaks, then the original function is called a smooth function. Smooth implies continuous, but not the other way around. There are functions that are continuous everywhere, yet nowhere differentiable. A smooth function can refer to a function that is infinitely differentiable.

$$
g(x)=e^{\cos (x+1)}
$$


Now we will see the accuracy of a hat function and a smooth function $e^{\cos (x+1)}$ using FFT.
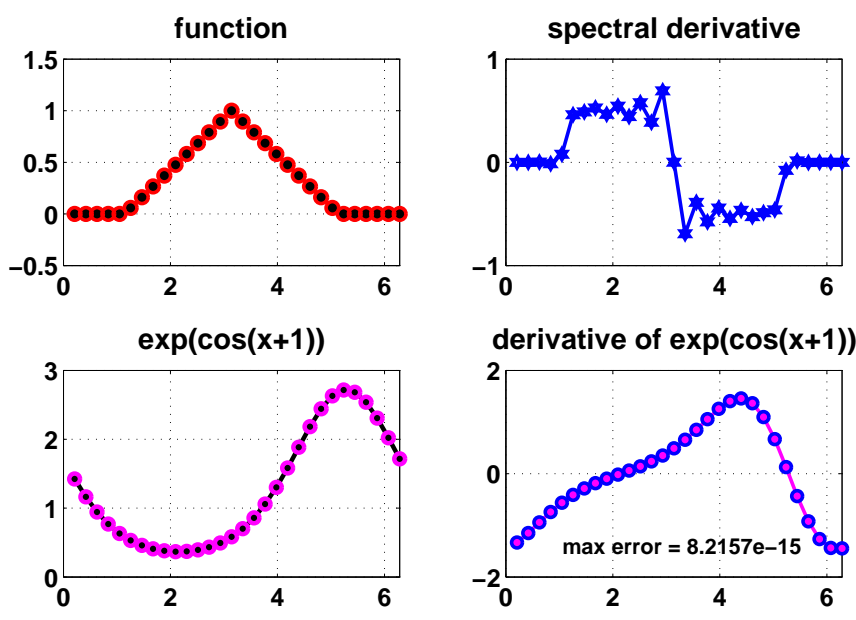

Figure 2.1: In the first row the graph of a hat function (2.6) and the corresponding derivative which is not so good. In the second row a smooth function (2.7) and the corresponding derivative which has error $8.217 \times 10^{-15}$.

Now, we consider the wave equation [12]

$$
\begin{cases}u_{t}+\gamma(x) u_{x}=0, & \text { for } x \in[0,2 \pi], t>0 \\ u(x, 0)=e^{-100(x-1)^{2}}, & \text { for } x \in[0,2 \pi]\end{cases}
$$

where, $\gamma(x)=\frac{1}{5}+\sin ^{2}(x-1)$.

The solution shows a beautiful wave propagation at variable speed. There is no spurious dispersion which is one of the conspicuous advantages of spectral methods.

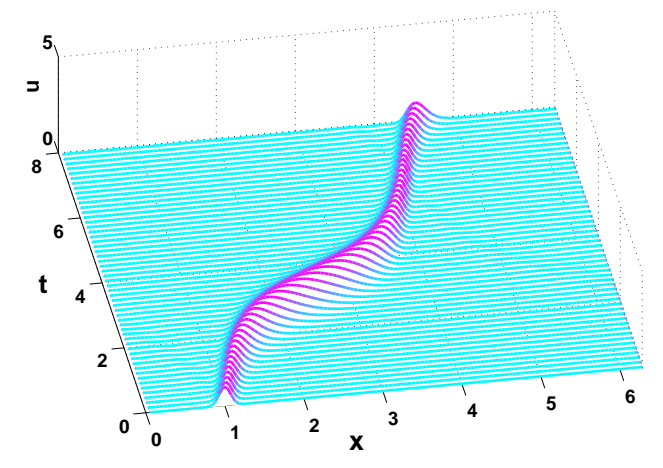

Figure 2.2: Solution of the wave equation.

\section{Chebyshev Points}

For non-periodic domains, algebraic polynomials on irregular grid are the right choice. The trigonometric basis fails to work for general non-periodic problems because of the failure of the requirement that the approximations $u_{N}(x, t)$ should converge rapidly to $u(x, t)$ as $N \rightarrow \infty$. Let we will work on $[-1,1]$ and the Chebyshev 
points are defined as

$$
x_{j}=\cos \left(\frac{j \pi}{N}\right), \quad j=1,2, \ldots, N .
$$

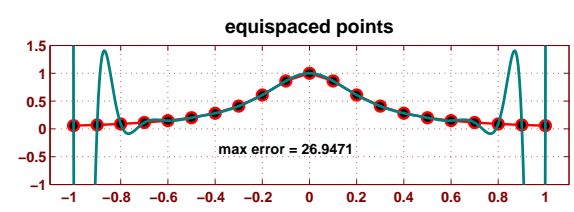

Chebyshev points

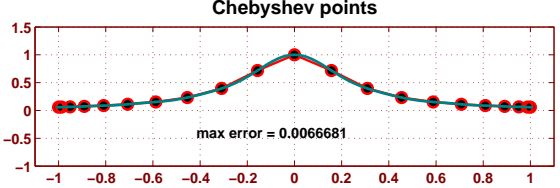

Figure 3.1: Polynomial interpolation for equispaced and Chebyshev points (3.1).
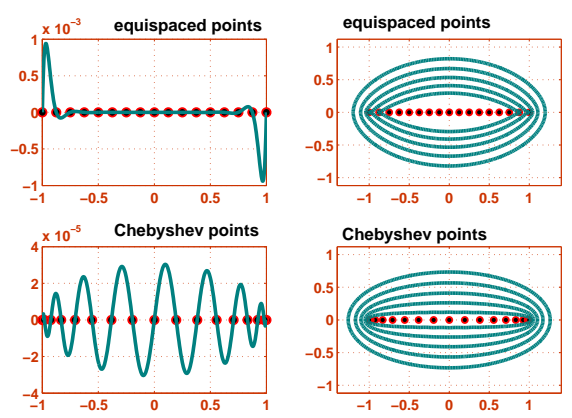

Figure 3.2: Polynomials and corresponding equipotential curves.
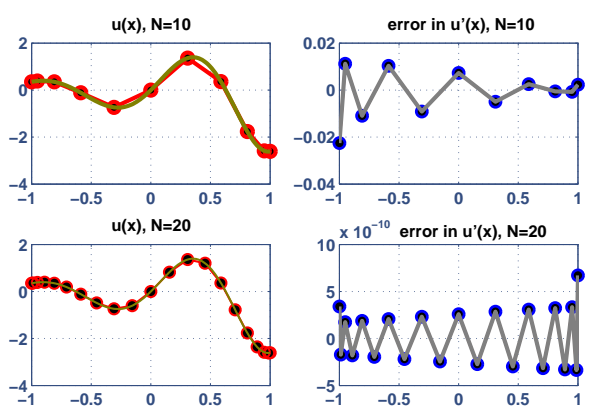

Figure 3.3: Polynomials with equispaced and Chebyshev roots of (3.3) and some level curves of the corresponding potentials in the complex plane.

For a given grid function $v$ defined on the Chebyshev points, we obtain a discrete derivative $w$ in two steps.

i. Let $p$ be the unique polynomial of degree $\leq n$ with $p\left(x_{j}\right)=v_{j}, 0 \leq j \leq n$.

ii. Set $w_{j}=p^{\prime}\left(x_{j}\right)$. 
This operation is linear and can be represented by an $(n+1) \times(n+1)$ matrix, which we shall denote by $D_{n}, \quad w=D_{n} v$. Here $n$ is an arbitrary positive integer even or odd. Using MATLAB program the differentiation matrix is given below:

$$
D_{2}=\left[\begin{array}{ccc}
3 / 2 & -2 & 1 / 2 \\
1 / 2 & 0 & -1 / 2 \\
-1 / 2 & 2 & -3 / 2
\end{array}\right] .
$$

Now we want to see how $D_{n}$ can be used to differentiate the smooth non-periodic function

$$
u(x)=e^{x} \sin (5 x)
$$

on grids with $n=10$ and $n=20$
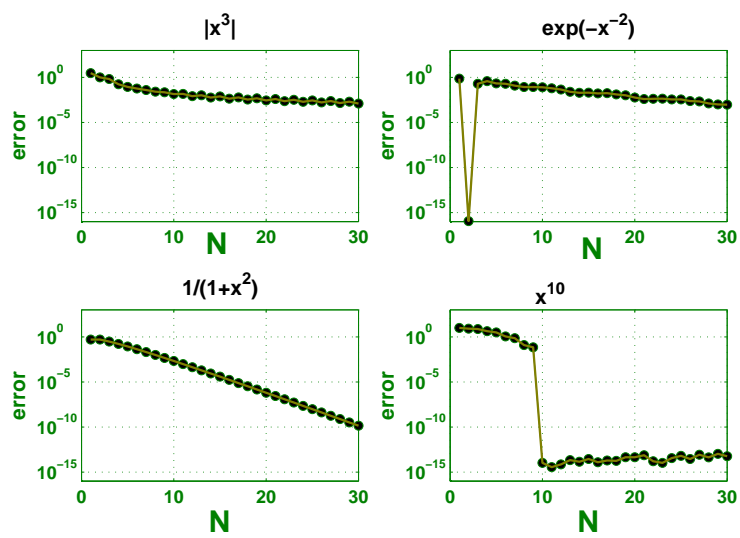

Figure 3.4: Accuracy of Chebyshev spectral differentiation.

Theorem 1. [3] (Composition formula) If $T_{n}(x)$ and $T_{m}(x)$ are Chebyshev polynomials $(m, n \geq 0)$ then

$$
\left(T_{m} \circ T_{n}\right)(x) \equiv T_{m}\left(T_{n}(x)\right)=T_{m n}(x)
$$

\section{Accuracy of the Method}

\subsection{Uniqueness}

Let $\Omega \subset R^{d}, d=2,3, \ldots$, be a bounded composition formula connected domain, with boundary $\Gamma=\partial \Omega$ and let $T$ be a positive real number. We recall the heat equation [4] as defined in the earlier section

$$
\begin{cases}u_{t}-\Delta u=f & \text { in } \Omega \times] 0, T[ \\ u=0 & \text { on } \partial \Omega \times] 0, T[ \\ u(\cdot, 0)=u_{0} & \text { in } \Omega .\end{cases}
$$

Where $f \in L^{2}(\partial \Omega \times] 0, T[)$ and $u_{0} \in L^{2}(\Omega)$ are given and $u$ is the unknown function. We find $u \in C^{0}\left(0, T ; L^{2}(\Omega)\right) \cap$ $L^{2}\left(0, T ; H_{0}^{1}(\Omega)\right)$ such that for all $\left.t \in\right] 0, T\left[\right.$ and $u(\cdot, 0)=u_{0}$ in $\Omega$

$$
\int \frac{\partial u}{\partial t}(x, t) v(x) \mathrm{d} x+\int \nabla u(x, t) \nabla v(x) \mathrm{d} x=\int f(x, t) v(x) \mathrm{d} x, \quad \forall v \in H_{0}^{1}(\Omega) .
$$

This problem has a unique solution $u \in C^{0}\left(0, T ; L^{2}(\Omega)\right) \cap L^{2}\left(0, T ; H_{0}^{1}(\Omega)\right)$. Now choosing $v=u$ in $(4.1)$ and integrating on $] 0, T[, 0 \leq t \leq T$, we deduce the following stability condition

$$
[u](t) \leq C\left(\left\|u_{0}\right\|_{L^{2}(\Omega)}^{2}+\|f\|_{L^{2}\left(0, t, H^{-1}(\Omega)\right)}^{2}\right)^{1 / 2} .
$$


Here $[\cdot]$ is a norm defined for $w \in L^{2}\left(0, T ; H_{0}^{1}(\Omega)\right)$ by

$$
[u](t)=\left(\|w\|_{L^{2}(\Omega)}^{2}+\|w\|_{L^{2}\left(0, t, H^{-1}(\Omega)\right)}^{2}\right)^{1 / 2} .
$$

And $C$ is a constant depending only on the domain $\Omega$. In the same way by taking $v=\frac{\partial u}{\partial t}$ and if $u_{0} \in H_{0}^{1}(\Omega)$, we obtain

$$
\|u(t)\|_{H^{-1}(\Omega)}^{2}+\left\|\frac{\partial u}{\partial t}\right\|_{L^{2}\left(0, T ; L^{2}(\Omega)\right)}^{2} \leq\left|u_{0}\right|_{H^{-1}(\Omega)}^{2}+\|w\|_{L^{2}(\Omega \times] 0, T[)}^{2} .
$$

Which implies that $u$ is in $L^{2}\left(0, t, H^{-1}(\Omega)\right)$.

\subsection{Time Discretization}

Let $T$ be a fixed positive number and $f$ be a function in $C\left(0, T, H^{-1}(\Omega)\right)$. We consider the partition of $[0, T]$ into $I$ equal sub-intervals $\left[t_{i-1}, t_{i}\right], 1 \leq i \leq I$ with $t_{0}<t_{1}<\cdots<t_{I}=T$ and $\delta t=t_{i}-t_{i-1}=T / I$. Using the implicit Euler scheme, the time discrete problem is written by

$$
u^{i}-u^{i-1}-\delta t \Delta u^{i}=\delta t f^{i} \text { in } \Omega, \quad 1 \leq i \leq I
$$

Actually we have to find $u^{i}$ in $L^{2}(\Omega) \times\left(H_{0}^{1}(\Omega)\right)^{I}, \quad 1 \leq i \leq I$ which satisfies

$$
u^{0}=u_{0} \text { in } \Omega
$$

and for each $v \in H_{0}^{1}(\Omega)$

$$
\int u^{i}(x) v(x) \mathrm{d} x+\delta t \int \nabla u^{i}(x) \nabla v(x) \mathrm{d} x=\int u^{i-1}(x) v(x) \mathrm{d} x+\delta t \int f^{i}(x) v(x) \mathrm{d} x .
$$

This problem has a unique solution $u^{i} \in H_{0}^{1}(\Omega), 1 \leq i \leq I$. Moreover by taking $v=u^{i}$ we obtain

$$
\left\|u^{i}\right\|_{L^{2}(\Omega)}^{2}+\delta t\left\|\nabla u^{i}\right\|_{L^{2}(\Omega)}^{2} \leq\left\|u^{i-1}\right\|_{L^{2}(\Omega)}^{2}+\left\|f^{i}(x)\right\|_{H^{-1}(\Omega)}^{2} .
$$

Summing with respect to $i$ we deduce the following stability condition on the solution $u^{i}, 1 \leq i \leq I$

$$
\left\|u^{i}\right\|_{L^{2}(\Omega)}^{2}+\delta t \sum_{l=1}^{i}\left\|u^{l}\right\|_{H^{-1}(\Omega)}^{2} \leq C\left(\left\|u^{0}\right\|_{L^{2}(\Omega)}^{2}+\delta t\left\|u^{l}\right\|_{H^{-1}(\Omega)}^{2}\right)
$$

where $C$ is a constant independent of $\delta t$.

\subsection{Spatial Discretization}

We suppose that $\Omega$ is a rectangle for $d=2$ or a parallelepiped for $d=3$. For a positive number $N$, we denote by $P_{N}(\Omega)$ the set of polynomials with $d$ variables and degree $\leq N$ for each variable. Let $\xi_{0}=-1$ and $\xi_{N}=1$ we define $(N-1)$ nodes $\xi_{j}, 1 \leq j \leq(N-1)$ and $(N+1)$ weights $\rho_{j}, 0 \leq j \leq N$ satisfying the Gauss-Lobatto quadrature formula on $]-1,1[$

$$
\int_{-1}^{1} \psi_{N} \mathrm{~d} x=\sum_{j=0}^{N} \psi_{N}\left(\xi_{j}\right) \rho_{j}, \quad \forall \psi_{N} \in P_{2 N-1}(]-1,1[) .
$$

We suppose that $u_{0}$ and $f$ respectively continuous on $\Omega$ and $\left.\Omega \times\right] 0, T[$. Using the Galerkin method and numerical integration the space discrete problem is written as: Find $u_{N}^{i} \in\left(P_{N}^{0}(\Omega)\right), 1 \leq i \leq I$ such that

$$
u_{N}^{0}=\zeta_{N}\left(u_{0}\right) \text { in } \Omega
$$


and for $1 \leq i \leq I$,

$$
\left(u_{N}^{i}, v_{N}^{i}\right)_{N}+\delta t\left(\nabla u_{N}^{i}, \nabla v_{N}^{i}\right)_{N}=\left(u_{N}^{i-1}, v_{N}\right)_{N}+\delta t\left(f^{i}, v_{N}\right)_{N}, \quad \forall v_{N} \in P_{N}^{0}(\Omega)
$$

here, $\zeta_{N}$ is the interpolation operator from $L^{2}(\Omega)$ to $P_{N}(\Omega)$.

Proposition 1. [2, 14] The above problem (4.6) and (4.8) has a unique solution $\left(u_{N}^{0}, u_{N}^{i}\right)_{1 \leq i \leq I}$ in $P_{N}(\Omega) \times$ $\left(P_{N}^{0}(\Omega)\right)^{i}$ and there exists a positive constant $C$ independent of $N$ such that

$$
\left\|u_{N}^{i}\right\|_{L^{2}(\Omega)}^{2}+\delta t \sum_{l=1}^{i}\left|u^{l}\right|_{H^{1}(\Omega)}^{2} \leq C\left(\left\|\zeta_{N}\left(u_{0}\right)\right\|_{L^{2}(\Omega)}^{2}+\delta t \sum_{l=1}^{i}\left\|\zeta_{N}\left(f^{l}\right)\right\|_{L^{2}(\Omega)}^{2}\right) .
$$

\subsection{Error Estimation}

Let $\Omega \subset R^{d}, d=2,3, \ldots$ be a bounded connected domain, with boundary $\Gamma=\partial \Omega$ and let $T$ be a positive real number. We consider the heat equation

$$
\begin{cases}u_{t}-\Delta u=f & \text { in } \Omega \times] 0, T[ \\ u=0 & \text { on } \partial \Omega \times] 0, T[ \\ u(\cdot, 0)=u_{0} & \text { in } \Omega\end{cases}
$$

where, $f \in L^{2}(\Omega \times] 0, T[)$ and $u_{0} \in L^{2}(\Omega)$ are given and $u$ is the unknown function. Now the error estimate between the solution $u$ of the continuous problem and the solution $u_{N}^{i}, 0 \leq i \leq I$ of the discrete problem will be established [4].

Theorem 2. [4] If $f$ and $u_{0}$ are respectively continuous on $\left.\Omega \times\right] 0, T[$ and $\Omega$ we have the following error estimates:

There exists a constant $C$ independent of $\delta t$ and $N$ such that

$$
\begin{aligned}
{\left[\mid u_{\delta t}-u_{N \delta t}\right]\left(t_{i}\right) \leq } & C\left(v_{N}^{l} \in \inf _{P_{N}(\Omega) \times P_{N-1}^{0}(\Omega)}\left(\left[\left|u_{\delta t}-u_{N \delta t}\right|\right]\left(t_{i}\right)+\left\|u_{0}-v_{N}^{0}\right\|_{L_{(\Omega)}^{2}}\right)\right. \\
& \left.+\left\|u_{0}-\mathfrak{J}_{N}\left(u_{0}\right)\right\|_{L_{(\Omega)}^{2}}+\left(\delta t \sum_{l=1}^{i}\left[\inf _{f_{N}^{l} \in P_{N-1}(\Omega)}\left\|f^{l}-\mathfrak{J} f^{l}\right\|_{L^{2}(\Omega)}\right]\right)^{1 / 2}\right) .
\end{aligned}
$$

\subsection{Accuracy using Fourier Transform}

Now we use the Fourier transform consisting of two steps. First, a smooth function decays rapidly and second, if the Fourier transform decays rapidly then the errors introduced by discretization are small. Here we will do it for the real line $R$. Smoothness of a function $u$ and decay of $\hat{u}$, Fourier transform are described in the following theorem.

Theorem 3. [15] Let $u \in L^{2}(R)$ have Fourier transform $\hat{u}$

i. If $u$ has $(p-1)$ continuous derivatives in $L^{2}(R)$ for some $p \geq 0$ and a $p^{\text {th }}$ derivatives of bounded variation, then $\hat{u}(k)=O|k|^{-p-1}$ as $|k| \rightarrow \infty$.

ii. If $u$ has infinitely many continuous derivatives in $L^{2}(R)$ then $\hat{u}(k)=O|k|^{-m}$ as $|k| \rightarrow \infty$. For every $m \geq 0$ the converse also hold.

iii. If there exist $a, c>0$ such that $u$ can be extended on an analytic function in the complex strip $|\operatorname{Im} z|<a$ with $\|u(\cdot+i y)\| \leq c$ uniformly for all $y \in(-a, a)$ where $\|u(\cdot+i y)\|$ is the $L^{2}$ norm along the horizontal line $\operatorname{Im} z=y$, then $u_{a} \in L^{2}(R)$, where $u_{a}(k)=e^{a|k|} \hat{u}(k)$. The converse also holds. 
iv. If $u$ can be extended to an entire function and there exist $a>0$ such that $|u(z)|=O\left(e^{a|z|}\right)$ as $z \rightarrow \infty$ for all complex values $z \in C$ then $\hat{u}$ has compact support in contained in $[-a, a]$, that is $\hat{u}_{k}=0$ for all $|k|>a$. The converse also holds.

This theorem quantifies our first argument that a smooth function has a rapidly decaying Fourier transform. We now turn to the second argument the connection between the decay of the Fourier transform and the accuracy of the band-limited interpolant. To make it easy we will consider the case of interpolation on the infinite grid $h z$.

Accuracy of the Fourier spectral differentiation is presented in the following statement.

Theorem 4. [16] Let $v \in L^{2}(R)$ have a $q^{\text {th }}$ derivative $(q \geq 1)$ of bounder variation and $w$ be the $q^{\text {th }}$ spectral derivative of $v$ on the grid $h z$.

1. If $v$ has $(p-1)$ continuous derivatives on $L^{2}(R)$ for some $p \geq q+1$ and a $p^{\text {th }}$ derivative of bounded variation, then $\left|w_{j}-v^{q}\left(x_{j}\right)\right|=O\left(h^{p-q}\right)$ as $h \rightarrow 0$.

2. If $v$ has infinitely many continuous derivatives in $L^{2}(R)$ then $\left|w_{j}-v^{q}\left(x_{j}\right)\right|=O\left(h^{m}\right)$ as $h \rightarrow 0$ for every $m \geq 0$.

3. If there exist $a, c>0$ such that $v$ can be extended to an analytic function in the complex strip $\mid$ Im $z \mid<a$ with $\|v(\cdot+i y)\| \leq c$ for all $y \in(-a, a)$ then $\left|w_{j}-v^{q}\left(x_{j}\right)\right|=O\left(h^{-\pi(a-\epsilon) / h}\right)$ as $h \rightarrow 0$ for every $\epsilon>0$.

4. If $v$ can be extended to an entire function and there exist $a>0$ such that for $z \in C|v(z)|=O\left(e^{a|z|}\right)$ as $|z| \rightarrow \infty$, then provided $h \leq \frac{\pi}{a}, w_{j}=v^{q}\left(x_{j}\right)$.

To check the accuracy, let us consider the following piecewise function:

$$
f(x)=\left\{\begin{array}{l}
|\cos (x+1)|^{3} \\
\exp \left(-\sin ^{-2}(x / 2)\right) \\
\left(1+\sin ^{2}(x / 2)\right)^{-2} \\
\sin (10 x)
\end{array}\right.
$$

Using MATLAB programming, various convergence rates are shown in the following figures.
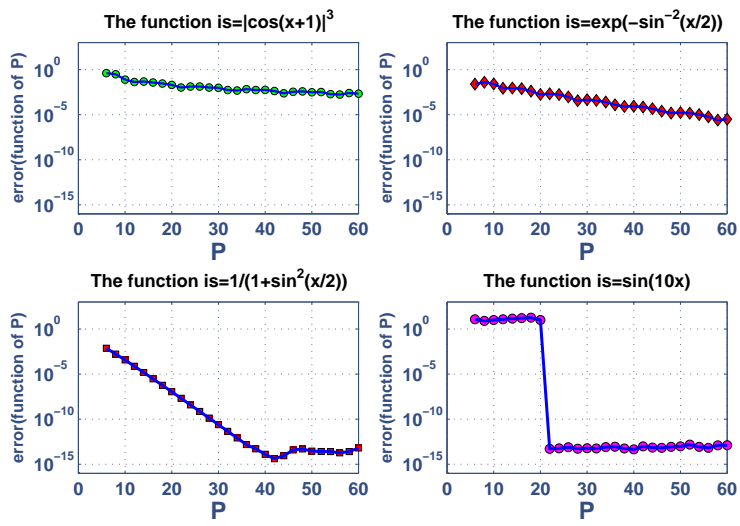

Figure 4.1: Error of four periodic functions (4.15). The smoother the function, the faster the convergence.

At last we want to give an example to illustrate spectral accuracy. We consider the following problem and observe the output.

$$
-v^{\prime \prime}+x^{2} v=\alpha v, \quad x \in \mathbb{R} \text { and } v \neq 0 .
$$




\begin{tabular}{|c|c|c|c|}
\hline $\mathrm{N}$ & Answer & $\mathrm{N}$ & Answer \\
\hline \multirow{3}{*}{$\mathrm{N}=6$} & 0.461472916995472 & & 0.978137281298610 \\
& 7.494134621050519 & $\mathrm{~N}=12$ & 3.171605320647192 \\
& 7.720916053006563 & & 4.455935291166793 \\
& 28.832483778340134 & & 8.924529058119939 \\
\hline \multirow{3}{*}{$\mathrm{N}=18$} & 0.999970001499297 & & 0.999999997629039 \\
& 3.000644066795820 & $\mathrm{~N}=24$ & 3.000000098410870 \\
& 4.992595324407724 & & 4.999997965273284 \\
& 7.039571897981499 & & 7.000024998156538 \\
\hline \multirow{3}{*}{$\mathrm{N}=30$} & 0.999999999999985 & & 0.999999999999942 \\
& 3.000000000000749 & $\mathrm{~N}=36$ & 3.000000000000017 \\
& 4.999999999975626 & & 5.000000000000001 \\
& 7.000000000508626 & & 7.000000000000019 \\
\hline
\end{tabular}

Table 1: Approximate four eigenvalues for 36 grid points.

This shows that the first four eigenvalues come out correct to 13 digits on a grid of just 36 points.

\section{Boundary Value Problems}

\subsection{Computational Algorithm}

Let $\Omega=(0, L)$. To accommodate the boundary conditions in the problem

$$
\begin{cases}u_{t}=u_{x x}+f(x, t), & (x, t) \in \Omega \\ u(x, t)=0, & (x, t) \in \partial \Omega \times(0, T) \\ u(x, 0)=u_{0}(x), & x \in \Omega .\end{cases}
$$

We take non-linearized eigenfunctions $w_{N}=\sin \left(\frac{n \pi x}{L}\right), n=1,2, \ldots$, as a basis of $H=L_{2}(\Omega)$. Let $S_{N}=$ $\operatorname{span}\left(w_{1}, w_{2}, \ldots, w_{N}\right)$ be a finite dimensional subspace of $V=H^{1}(\Omega)$. Since $S_{N}$ is closed subspace of Hilbert space, $S_{N}$ itself is a Hilbert space. Thus we define a natural projection $P_{N}$ from $H$ onto $S_{N}$ in $H$. Let $u_{N}(t)=u_{N}(\cdot, t) \in S_{N}$ that satisfies

$$
\left\{\begin{array}{l}
\left(\frac{\partial u_{N}}{\partial t}, v\right)+\left(u_{N}, v\right)=(f, v), \quad t \in(0, T) \\
\left(u_{N}(0)-u(0), v\right)=0, \quad \forall v \in S_{N} .
\end{array}\right.
$$

To discuss the computational algorithm to find solutions $u_{N}$, let $\left\{w_{j}\right\}_{j=1}^{\infty}=\left\{\sin \frac{j \pi x}{L}\right\}_{j=1}^{\infty}$ be eigenfunctions of $A$ that forms a basis in $H$. Then $\left\{w_{j}\right\}_{j=1}^{\infty}$ is a basis on $v$. We fix $N \in \mathcal{N}$. Let $V_{N}=\operatorname{span}\left(w_{1}, w_{2}, \ldots, w_{N}\right)$. Let $P_{N}: H \rightarrow V_{N}$ be the projection operator defined by $P_{N} v=\sum_{j=1}^{N}\left(v, w_{j}\right) w_{j}$ for any $v \in H$. Then the solution $u_{N}$ is given by

$$
u_{N}(x, t)=\sum_{j=1}^{N} g_{j} N(t) w_{j}(x)
$$

which satisfies

$$
\begin{aligned}
& \frac{\mathrm{d}}{\mathrm{d} t}\left(u_{N}, w_{j}\right)+a\left(u_{N}, w_{j}\right)=\left(f, w_{j}\right) \\
& u_{N}(0)=P_{N} u_{0}, \quad \forall j .
\end{aligned}
$$

Let $\bar{g}_{N}=\left\{g_{j N}\right\}_{j=1}^{N} \in R^{N}$. We can write the following vector differential equation

$$
\bar{g}_{N}^{\prime}(t)+\Lambda \bar{g}_{N}(t)=\bar{F}(t, x)
$$


with the initial data

$$
\bar{g}_{N}(0)=\left[\begin{array}{c}
\left(P_{N} u_{0}, w_{1}\right) \\
\left(P_{N} u_{0}, w_{2}\right) \\
\vdots \\
\left(P_{N} u_{0}, w_{N}\right)
\end{array}\right],
$$

where $u_{0} \in L^{2}(0, T ; V), g_{N}(t) \in R^{N}, F\left(t, g_{N}\right) \in R^{N}$.

In the rest of the section, the discussion is about how matrices can be used to solve some boundary value problems in both ordinary and partial differential equations.

Example 1. We consider the linear ordinary differential equation boundary value problem

$$
u_{x x}=e^{2 x} ; \quad x \in(-1,1), \quad u(1)=0=u(-1) .
$$

To solve the problem numerically, we can compute the second derivative $D_{N}^{2}$ by squaring $D_{N}$. For simplicity we will square $D_{N}$ to get $D_{N}^{2}$.

For this problem we can proceed as follows. We take the interior Chebyshev points $x_{1}, x_{2}, \ldots, x_{N-1}$ as computational grid, with $v=\left(v_{1}, v_{2}, \ldots, v_{N-1}\right)^{T}$ as the corresponding vector of unknowns. Then the spectral differentiation carried out like this:

$i$. Let $p(x)$ be the unique polynomial of degree $\leq N$ with $p( \pm 1)=0$ and $p\left(x_{j}\right)=v_{j}, 1 \leq j \leq N-1$.

ii. Set $w_{j}=p^{n}\left(x_{j}\right), 1 \leq j \leq N-1$.

Now $D_{N}^{2}$ is an $(N+1) \times(N-1)$ matrix that produce a vector $\left(v_{0}, v_{1}, \ldots, v_{N}\right)^{T}$ and its turn to a vector $\left(w_{0}, w_{1}, \ldots, w_{N}\right)^{T}$ such that first fix $v_{0}$ and $v_{N}$ at zero and then ignore $w_{0}$ and $w_{N}$. This implies that the first and last columns of $D_{N}^{2}$ have no effect and the first and last rows have no effect.

$$
\tilde{D_{N}^{2}}=D_{N}^{2}(1: N-1,1: N-1)
$$

Since indices starts at 1 instead of 0 , this will become $D_{N}^{2}=D_{N}^{2}(2: N, 2: N)$. With $\tilde{D_{N}^{2}}$ in hand, the numerical solution of becomes a matter of solving a linear system of equations:

$$
\tilde{D_{N}^{2}} v=f
$$
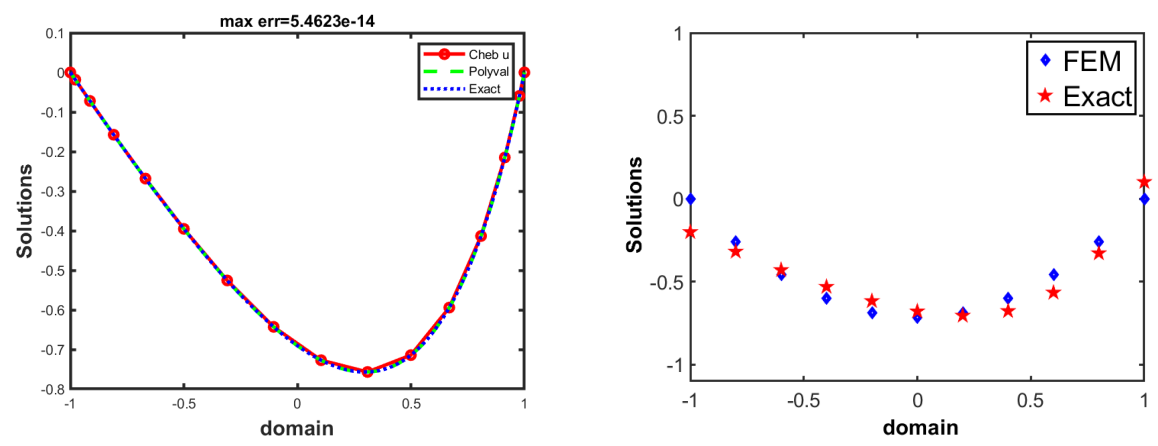

Figure 5.1: Solution of the linear boundary value problem (5.4), (left) Spectral method, (right) Finite element method.

The figure 5.1 presents the solutions using spectral and finite element methods comparing with exact solutions. It is seen from the left diagram of figure 5.1 that visibly exact and numerical solutions coincide while in right diagram of figure 5.1, the approximate and exact solutions are very closer to each other. The results ensure the higher accuracy of proposed spectral method. 
Example 2. Suppose we change the previous problem to

$$
u_{x x}=e^{u}, \quad x \in(-1,1), \quad u(1)=0=u(-1) .
$$

For this, we choose an initial guess, such as the vector zeros, and then iterate by repeatedly solving the system of equations:

$$
\tilde{D_{N}^{2}} v_{n e w}=e^{v_{\text {old }}}
$$

where, $e^{v}$ is the column vector defined by $e^{v_{j}}$. Using a MATLAB program which implements this iteration with a crude stopping criterion and convergence occurs in 29 steps. To confirm the correct solution, we print results for various $N$ in the following table:

\begin{tabular}{|c|c|c|}
\hline$N$ & Iteration Number & $u(0)$ \\
\hline 2 & 34 & -0.35173371124920 \\
\hline 4 & 29 & -0.36844814823915 \\
\hline 6 & 29 & -0.36805450387666 \\
\hline 8 & 29 & -0.36805614384219 \\
\hline 10 & 29 & -0.36805602345302 \\
\hline 12 & 29 & -0.36805602451189 \\
\hline 14 & 29 & -0.36805602444069 \\
\hline 16 & 29 & -0.36805602444149 \\
\hline 18 & 30 & -0.36805602444143 \\
\hline 20 & 29 & -0.36805602444143 \\
\hline
\end{tabular}

Table 2: Approximate results by spectral method with different iteration numbers.
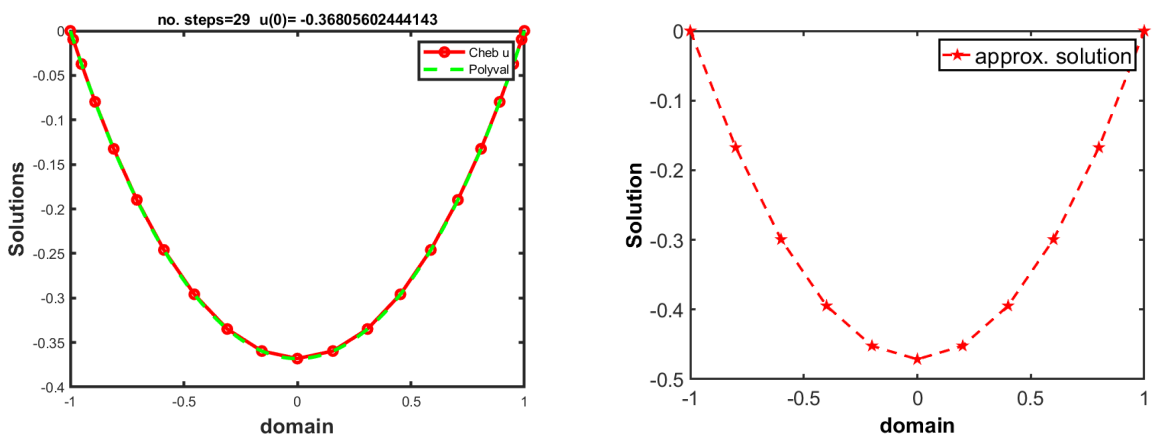

Figure 5.2: Solution of the non-linear boundary value problem (5.5), (left) Spectral method, (right) Finite element method.

The results in figure 5.2 depicted the solutions of non-linear problem (5.5). Observed that within 29 steps the result is pretty much accurate, as presented in left side of figure 5.2. The right figure is designed using the finite element method.

Example 3. As a third application of the modified second order differentiation matrix $\tilde{D_{N}^{2}}$, consider the eigenvalue boundary value problem:

$$
u_{x x}=\lambda u, \quad x \in(-1,1), \quad u(1)=0=u(-1) .
$$




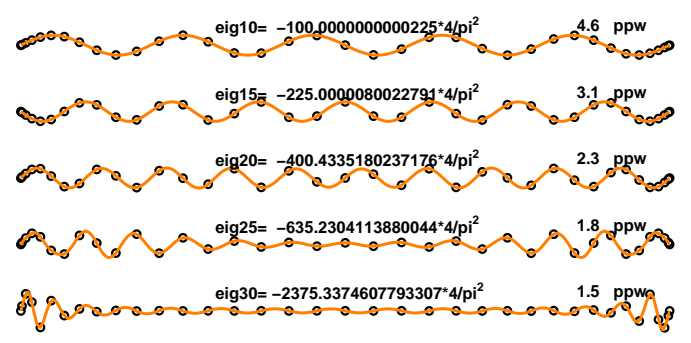

Figure 5.3: Eigenvalue and eigenmodes of $\tilde{D_{N}^{2}}$ and the number of grid points per wave length at the center of the grid.

The eigenvalue of this program are $\lambda=-\frac{\pi^{2} n^{2}}{4 n}=1,2, \ldots$ with corresponding eigenfunctions $\sin \left(\frac{n \pi(x+1)}{2}\right)$. The number of plots of figure 5.3 reveal a great deal about the accuracy of spectral methods. Eigenvalues 5,10 and 15 are obtained too many digits of accuracy, and eigenvalue 20 is still pretty good. Eigenvalue 25 is accurate to only one digit and eigenvalue 30 is wrong by a factor of 3 . The quantity that explains this behavior is the number of points per wavelength in the central, coarsest part of the grid near $x=0$. With at least 2 points per wavelength, the grid is fine enough to resolve the wave. With less than 2 points per wavelength, the wave cannot be resolved, and eigenvectors are obtained that are meaningless as approximations to the original problem.

Finally in the last example, we will discuss how to extend these methods to boundary value problems in 2D.

Example 4. [6, 13] A variation of the Poisson equation is the Helmholtz equation

$$
-u_{t t}+u_{x x}+u_{y y}=e^{i k t} f(x, y), \quad x, y \in(-1,1), \quad U=0 .
$$

After separation of variables to $U(x, y, t)=e^{i k t} u(x, y)$, we solve this problem for the particular choices

$$
k=9, \quad f(x, y)=e^{\left(-10\left[(y-1)^{2}+(x-1 / 2)^{2}\right]\right)} .
$$

The solution appears as a mesh plot in the left figure of 5.4 and as a contour plot in right of 5.4. It is clear that the response generated by this forcing function $f(x, y)$ for this value $k=9$ has approximately the form of a wave with three half-wavelengths in the $x$ direction and five half-wavelengths in the $y$ direction. Such a wave in an eigenfunction of the homogeneous Helmholtz problem with eigenvalue

$$
k=\frac{1}{2} \sqrt{3^{2}+5^{2}} \approx 9.1592
$$

Here, $k=9$ gives near-resonance with this $(3,5)$ mode.
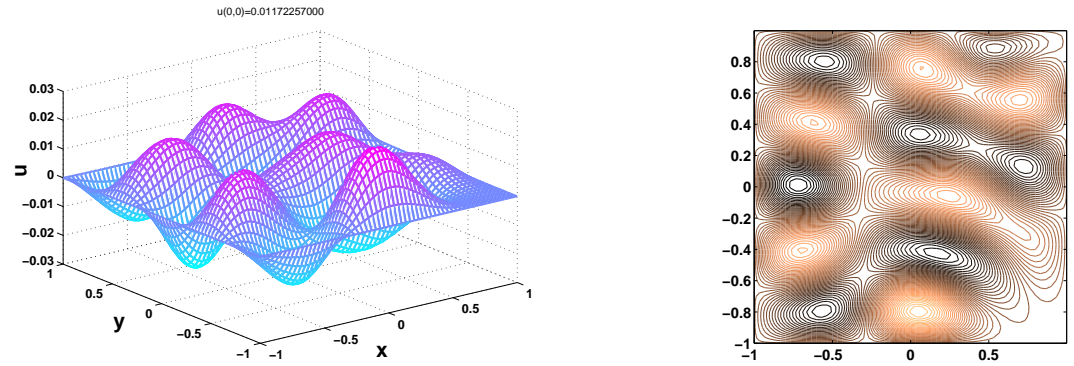

Figure 5.4: Solution of the Helmholtz problem (5.7). The computed value $u(0,0)$ is accurate to nine digits. Same result is represented as contour plot in right diagram. 


\section{Concluding Remarks}

In the present paper, we have presented a highly accurate spectral collocation method for solving ordinary and partial differential equations with homogeneous and non-homogeneous Dirichlet boundary conditions. It is developed by using Fourier transform. Its detail derivation is discussed along with its error estimations and stability analysis. Then we apply our proposed method in different problems. From the numerical and graphical results of those problems, we can conclude that spectral collocation method in an effective, efficient, stable method with higher accuracy and less computational cost.

However, the spectral methods have several limitations. Coding is more complex and challenging. To implement the results, the methods are more complicated. The method use basis global function, not well suited for handling localized features and/or sharp gradients; remember the Gibbs phenomenon while FEM and those based on local basis functions usually do better. Spectral methods are expensive at high resolutions. The operation count for FFT is proportional to $N \ln (N)$ instead of $N$ as for grid point method - one of the reasons why grid point method is coming back in fashion for global models. In addition, few regional model uses spectral method because of the lack of natural periodic boundary condition [17]. Moreover, a poorly designed spectral method may perform much worse than simpler finite difference or finite element techniques.

\section{References}

[1] Gottlieb, D., Hussaini, M. Y., Orszag, S. A., (1983). Theory and Applications of Spectral Methods, NASA Langley Research Center, Hainpton, Virginia.

[2] Canuto, C., Hussaini, M.Y., Quarteroni, A., Zang, T.A., (2007). Spectral methods: evolution to complex geometries and applications to fluid dynamics, Springer Science \& Business Media.

[3] Trefethen, L.N., (2000). Spectral Methods in MATLAB, SIAM, Philadelphia, PA.

[4] Dutykh, D., (2016). A Brief Introduction to Pseudo-Spectral Methods, Cornell University.

[5] Thapa, N., Gudejko, M., (2014). Numerical Solution of Heat Equation by Spectral Method, Applied Mathematical Sciences 8, p. 397-404.

[6] Morton, K.W., Mayers, D., (2005). Numerical Solution of Partial Differential Equations, Cambridge University Press 2.

[7] Canuto, C., Hussaini, M.Y., Quarteroni, A.M., Zang, J.T.A., (1988). Spectral Method in Fluid Dynamics, Springer Verleg.

[8] Kumar, B.V.R., Mehra, M., (2005). A wavelet-Taylor Galerkin method for parabolic and hyperbolic partial differential equations, International Journal of Computational Methods 2, p. 75-97.

[9] Mehra, M., Kumar, B.V.R., (2005). Time-accurate solution of advection-diffusion problems by waveletTaylor-Galerkin method, Communications in numerical methods in engineering 21, p. 313-326.

[10] Mehra, M., (2009). Wavelets and Differential Equations-A short review, In AIP Conference Proceedings, American Institute of Physics 1146, p. 241-252.

[11] Burden, R.L., Faires, J.D., (2010). Numerical analysis, Brooks/Cole, USA.

[12] Kanth, ASVR., Aruna, K., (2009). Differential transform method for solving the linear and nonlinear Klein-Gordon equation, Computer Physics Communications 180, p. 708-711.

[13] Carpenter, M.H., Gottileb, D., (1996). Spectral methods on arbitrary grids, Journal of Computational Physics, ELSEVIER 129, p. 74-86 
[14] Hesthaven, J.S., (2000). Spectral penalty methods, Applied Numerical Mathematics, ELSEVIER 33, p. $20-41$.

[15] Korner, T.W., (1990). Fourier Analysis, Cambridge University Press.

[16] Hille, E., (1962). Analytic Function Theory, Chelsea.

[17] Juang, H. M. H., and Kanamitsu, M. (1994). The NMC regional spectral model, Mon. Wea. Rev., 122, p. $3-26$. 